\title{
Alternative new notation for quantum information theory
}

\author{
Chong Li* ${ }^{*}$ He-Shan Song, and Ling Zhou \\ Department of physics, \\ Dalian University of Technology, \\ Dalian City, 116023 P.R.China
}

May

\begin{abstract}
A new notation has been introduced for the quantum information theory. By this notation,some calculations became simple in quantum information theory such as quantum swapping, quantum teleportation.
\end{abstract}

\section{Introduction}

Since the concept of entanglement[1] was introduced into quantum physics, it plays an important roles in quantum information theory. Nearly all of quantum information processes are based on entangled state such as quantum code[2], quantum swapping[3], quantum teleportation[4] and so on.

In the quantum information theory some calculations are very complex. For example, in quantum teleportation we must write out all the joint states, and need to find out the relation between the Alice's joint Bell states measured results and the receiver Bob's local operation for his particle. Furthermore, if we perform a teleptortation of $n$-dimension state, the calculations are tremendous! Evenmore we make a series of 2-dimensional quantum entanglement swapping, the calculations are very difficult.

*lichong@student.dlut.edu.cn 
We have introduced a kind of notation[11], which was represented in Ref.[10], for quantum teleportation. In that paper, we give a powerful theorem for general teleportation, by which one can easily construct Bob's operation.

In this paper we generalize the notation to much more quantum information process. By using this tool,we give a new criteria for Schmidt decomposition of entangled state. In terms of this criteria we need not to calculate partial inner-product for the joint density operator.

This notation can simplify calculation of quantum swapping include series quantum swapping,

As we know, notation is often used in physics, it can simplify some calculation just as Dirac sign does. Here we try to make following notation for quantum information.

In the paper[11],we introduced a notation for quantum teleportation, which is defined as follows,

Let $\mathbf{h}$ be a finite Hilbert space, and $\operatorname{dim}(\mathbf{h})=N$, so 2-partite Hilbert space $\mathbf{H}=h \otimes h$.

We definite some useful notations :

Definition $1 \boldsymbol{\alpha}=\left(\begin{array}{llll}\alpha_{0} & \cdots & \alpha_{N-2} & \alpha_{N-1}\end{array}\right)$,

Definition $2 \mathbf{e}_{i}=\left(\begin{array}{llll}|0\rangle_{i} & \cdots & |N-2\rangle_{i} & |N-1\rangle_{i}\end{array}\right)$,

Definition $3 \tilde{\mathbf{e}}_{i}=\left(\begin{array}{lll}{ }_{i}\langle 0| & \cdots & { }_{i}\langle N-2| \\ \langle & \langle\langle N-1|\end{array}\right)$.

Let set $\left\{\begin{array}{llll}|0\rangle_{i} & \cdots & |N-2\rangle_{i} & |N-1\rangle_{i}\end{array}\right\}$ be a group of orthonormal complete basis of the $i$-th Hilbert space $\mathbf{h}_{i}$. So an arbitrary state $|\varphi\rangle=\sum_{i=0}^{N-1} \alpha_{i}|i\rangle$ can be written as $|\varphi\rangle=\boldsymbol{\alpha e}^{t}$ (where superscrip $t$ means transposition). Arbitrary bipartite state $\left|\psi_{12}\right\rangle=\sum_{i j} a_{i j}|i\rangle_{1}|j\rangle_{2}$ can be written as $\left|\psi_{12}\right\rangle=$ $\mathbf{e}_{1} \mathbf{A} \mathbf{e}_{2}^{t}=\mathbf{e}_{2} \mathbf{A}^{t} \mathbf{e}_{1}^{t}$, where $\mathbf{A}$ is a known matrix $\mathbf{A}_{i j}=a_{i j}$ and $A^{t}$ is the transposed of $A$. If we perform a local operation $U_{1}$ on particle 1 , the state become $U_{1}\left|\psi_{12}\right\rangle=\mathbf{e}_{1} U_{1} \mathbf{A} \mathbf{e}_{2}^{t}$, and it is $U_{2}\left|\psi_{12}\right\rangle=\mathbf{e}_{1} \mathbf{A} U_{2}^{t} \mathbf{e}_{2}^{t}$ with local operation $U_{2}$.

We will find the merits of this notation from the following discussion. 


\section{Application of the notation for entangle- ment research}

The phenomenon of entanglement is a remarkable feature of quantum theory. It play a crucial role in the discussions of quantum mechanics and quantum information theory. The entangled states are important for quantum information theory. In particular, the pure bipartite entangled state (PBES) is used in most quantum information transported process such as quantum teleportation, quantum code et al. So how to find out the PBES from PBS(pure bipartite state) is important. As we know, any pure bipartite entangled state can be expressed as Schmidt decomposition, so the criteria for pure bipartite entangled state is, if the Schmidt number of the pure bipartite state is more than one. by the notation, a pure bipartite state $\left|\psi_{12}\right\rangle$ can be described as

$$
\begin{aligned}
\left|\psi_{12}\right\rangle & =\sum_{i j} a_{i j}|i\rangle_{1}|j\rangle_{2} \\
& =\left(|0\rangle_{1}|1\rangle_{1} \cdots|n-1\rangle_{1}\right) A\left(|0\rangle_{2}|1\rangle_{2} \cdots|n-1\rangle_{2}\right)^{t} \\
& =\mathbf{e}_{1} \mathbf{A e}_{2}^{t} .
\end{aligned}
$$

Where all entangled information are included in the matrix $A$. Here we proposed a criteria for this.

Criteria 1 : The state $\left|\psi_{12}\right\rangle$ is entangled state, if and only if there are more than one non-zero eigenvalues of the matrix $A A^{\dagger}$, the number of the non-zero eigenvalues is just Schmidt number.

Proof:As we know, any bipartite entangled state can be written as Schmidt decomposition, namely

$$
\left|\psi_{12}\right\rangle=\sum_{i j} a_{i j}|i\rangle_{1}|j\rangle_{2}=\sum_{k} \sqrt{P_{k}}|\widetilde{k}\rangle_{1}|\widetilde{k}\rangle_{2}
$$

the joint density operator is

$$
\rho\left(\psi_{12}\right)=\sum_{i j l m} a_{i j}\left(a_{l m}\right)^{\dagger}|i\rangle_{1}|j\rangle_{2}\left\langlem | _ { 2 } \left\langle\left. l\right|_{1}\right.\right.
$$

and the density operator of the qubit 1 is 


$$
\begin{aligned}
\rho_{1}\left(\psi_{12}\right) & =\sum_{i l m} a_{i m}\left(a_{l m}\right)^{\dagger}|i\rangle_{1}\left\langle\left. l\right|_{1}\right. \\
& =A A^{\dagger}=B
\end{aligned}
$$

We can calculate out the eigenvalues of $\rho_{1}\left(\psi_{12}\right)$

$$
\operatorname{det}(\lambda-B)=0 \Longrightarrow \boldsymbol{\lambda}
$$

where $\boldsymbol{\lambda}=\left(\lambda_{1} \lambda_{2} \cdots \lambda_{n}\right)$ and Schmidt number $N_{s} \geqslant 2$ then the state $\left|\psi_{12}\right\rangle$ is entangled state, namely there are more than two non-zero eigenvalue of the state $\left|\psi_{12}\right\rangle$.

$$
|\widetilde{k}\rangle_{1}=\sum_{j} \dot{t}_{k j}|j\rangle_{1} \text { and } \rho_{1}\left(\psi_{12}\right)|\widetilde{k}\rangle_{1}=\lambda_{k}|\widetilde{k}\rangle_{1}
$$

Let $\left|\psi_{12}\right\rangle=\sum_{k} \sqrt{\lambda_{k}}|\widetilde{k}\rangle_{1}|\widetilde{k}\rangle_{2}$, where $|\widetilde{k}\rangle_{2}=\sum_{i} \alpha_{i}^{k}|i\rangle_{2}$ with ${ }_{2}\langle\widetilde{k} \mid \widetilde{j}\rangle_{2}=$ $\delta_{k j}$, considered $\left|\psi_{12}\right\rangle=\sum_{i j} a_{i j}|i\rangle_{1}|j\rangle_{2}$, we can get all $\alpha_{i}^{k}$. So $|\widetilde{k}\rangle_{2}$ is known. $\left|\psi_{12}\right\rangle=\sum_{k} \lambda_{k}|\widetilde{k}\rangle_{1}|\widetilde{k}\rangle_{2}$

From this criteria we not only can get the Schmidt number of the state, but also can find out the Schmidt decomposition expression for the state.

Criteria 2 : The state $\left|\psi_{12}\right\rangle$ is entangled state, if and only if the rank of the matrix $A$ is more than one, it is just the Schmidt number.

Proof:As we know, the non-zero eigenvalue number of any matrix is equal to its rank.

There must be matrix $T, T T^{\dagger}=M_{i i} \delta_{i j}$ with $M_{i i} \in\{0,1\}$

$$
T A T^{\dagger}=K, \text { with } K_{i j}=a_{i j} \delta_{i j}
$$

and

$$
T A^{\dagger} T^{\dagger}=K^{\dagger}, \text { with } K_{i j}^{\dagger}=b_{i j} \delta_{i j}
$$

So

$$
T \rho_{1} T^{\dagger}=T A T^{\dagger} T A^{\dagger} T^{\dagger}=K K^{\dagger}=\sigma_{i j} \delta_{i j}
$$

with $\sigma_{k k}=a_{k k} b_{k k}$. finally we can easily get 


$$
R\left(\rho_{1}\right)=R(A)=R\left(A^{\dagger}\right) .
$$

\section{Application for quantum swapping and bi- partite state quantum teleportation}

Quantum information attracted more attention since quantum teleportation has been proposed. Quantum swapping is important in quantum information. But there are some complicated calculation in quantum cryptography[14] and quantum swapping, furthermore multi-dimension teleportation or series of swapping, while by this notation, all of this kinds of calculation become easy.

\subsection{Quantum swapping}

As we know, any quantum information process is based on entangled state. If sender and receiver do not share any common entangled state, how they to communicate each other? The answer is that they can make a quantum swapping through another person as follow. Alice (sender) has a entangled states $\left|\phi_{12}\right\rangle$, Bob(receiver) has a entangled states $\left|\varphi_{34}\right\rangle$, Alice send qubit 2 to Charley(the third person) and $B o b$ send a qubit 3 to him, then Charley make a joint measurement on qubit 2 and 3, he told Alice and Bob his measured result, then Alice and Bob share a new entangled state $\left|\psi_{14}\right\rangle$, this process is quantum swapping.

$$
\begin{aligned}
& \left|\phi_{12}\right\rangle=\left(|0\rangle_{1}|1\rangle_{1} \cdots|n-1\rangle_{1}\right) A\left(|0\rangle_{2}|1\rangle_{2} \cdots|n-1\rangle_{2}\right)^{t} \\
& \left|\varphi_{34}\right\rangle=\left(|0\rangle_{3}|1\rangle_{3} \cdots|n-1\rangle_{3}\right) C\left(|0\rangle_{4}|1\rangle_{4} \cdots|n-1\rangle_{4}\right)^{t}
\end{aligned}
$$

measured result

$$
\left|\phi_{23}^{\prime}\right\rangle=\left(|0\rangle_{2}|1\rangle_{2} \cdots|n-1\rangle_{2}\right) B\left(|0\rangle_{3}|1\rangle_{3} \cdots|n-1\rangle_{3}\right)^{t}
$$

so the new entangled state 


$$
\begin{aligned}
& \left|\psi_{14}\right\rangle=\left\langle\phi _ { 2 3 } ^ { \prime } \left|\left(\left|\phi_{12}\right\rangle \otimes\left|\varphi_{34}\right\rangle\right)\right.\right.
\end{aligned}
$$

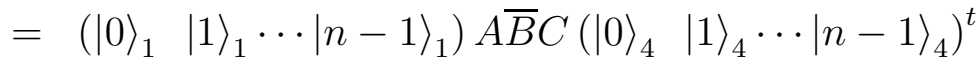

$$
\begin{aligned}
& =\rho\left(|0\rangle_{1}|1\rangle_{1} \cdots|n-1\rangle_{1}\right) F\left(|0\rangle_{4}|1\rangle_{4} \cdots|n-1\rangle_{4}\right)^{t}
\end{aligned}
$$

where

$$
\begin{gathered}
\left|\psi_{14}\right\rangle=\left(|0\rangle_{1} \quad|1\rangle_{1} \cdots|n-1\rangle_{1}\right) F\left(|0\rangle_{4}|1\rangle_{4} \cdots|n-1\rangle_{4}\right)^{t} \\
F=A \bar{B} C / \rho \cdot\left(\bar{B}_{i j}=B_{i j}^{*}\right) \\
\rho=\sqrt{\operatorname{tr}\left\{(A \bar{B} C)(A \bar{B} C)^{\dagger}\right\}}
\end{gathered}
$$

Further more, there are $n$ bipartite entangled states $\left\{\left|\phi_{12}\right\rangle\left|\phi_{34}\right\rangle \cdots\left|\phi_{(2 n-1) 2 n}\right\rangle\right\}$ and $\left|\phi_{(2 k+1)(2 k+2)}\right\rangle=\mathbf{e}_{2 k-1} \mathbf{A}_{k} \mathbf{e}_{2 k}^{t}\left|\phi_{(2 k)(2 k+1)}^{\prime}\right\rangle=\mathbf{e}_{2 k} \mathbf{B}_{k} \mathbf{e}_{2 k+1}^{t}$, then through $n-1$ quantum swappings the final entangled state of particle 1 and particle $2 n$ is $\left|\psi_{1(2 n)}\right\rangle=\mathbf{e}_{1} \mathbf{F e}_{2 n}^{t}$, where

$$
\mathbf{F}=\left(\prod_{k=1}^{n-1} \mathbf{A}_{k} \overline{\mathbf{B}}_{k}\right) \mathbf{A}_{n}
$$

In fact, quantum communication can be realized by sending one of the entanglement pairs, while during long way transformation the entanglement will decrease and some information will be losted. However we can overcome this problem by a series quantum swapping as shown in the Fig 1 (where $A_{k}$ means the $k$-th EPR pairs, and the $B_{k}$ is the $k$-th joint measerment). The calculation of the series quantum swapping is very complex even $n$ is a large number, but by the notation, the calculation become concise. 


\subsection{Bipartitie state teleportation}

Earlier studies have been confined to the teleportation of single-body quantum states [4], which is only a special case. Recently teleportation of multibody quantum states was attracted more attentions[12-13], the studies are only confined to teleportation of bipartite entangled state. In this paper we study on how to teleport a bipartite states by two EPR states based by the notation[11], and construct Bob's local operation.

Supposed that Alice was going to teleport a bipartite state $|\phi\rangle_{12}=$ $\sum_{i j} A_{i j}|i\rangle_{1}|j\rangle_{2}=\vec{e}_{1} A \vec{e}_{2}^{t}$ to $B o b$, she shared two known entanglement states $|\alpha\rangle_{34}=\vec{e}_{3} B \vec{e}_{4}^{t}$ and $|\beta\rangle_{56}=\vec{e}_{5} C \vec{e}_{6}^{t}$ with Bob. Alice performed a joint states measurement on particle 2 and particle 3 , the result $|\delta\rangle_{23}=\vec{e}_{2} D \vec{e}_{3}^{t}$, by the eq.(1) we get the state of particle 1 and particle $4|\gamma\rangle_{14}=\vec{e}_{1} M \vec{e}_{4}^{t}=$ $\vec{e}_{4} M^{t} \vec{e}_{1}^{t}$, where $M=\frac{A \bar{D} B}{\rho_{1}}$. Then Alice made another joint states measurement on particle 1 and particle 5 , the result $|\eta\rangle_{15}=\vec{e}_{1} F \vec{e}_{5}^{t}$, by the eq.(1) the state of particle 4 and particle $6|\varphi\rangle_{46}=\vec{e}_{4} N \vec{e}_{6}^{t}$ was easily obtained, where $N=\frac{M^{t} \bar{F} C}{\rho_{2}}=\frac{B^{t} \bar{D}^{t} A^{t} \bar{F} C}{\rho_{1} \rho_{2}}$.

$$
|\varphi\rangle_{46}=|\varphi\rangle_{64}=\vec{e}_{4} \frac{B^{t} \bar{D}^{t} A^{t} \bar{F} C}{\rho_{1} \rho_{2}} \vec{e}_{6}^{t}=\vec{e}_{6} \frac{A \bar{D} B \bar{F} C}{\rho_{1} \rho_{2}} \vec{e}_{4}^{t}
$$


Alice told $B o b$ her joint states measurements, $B o b$ perform a unitary operator $U_{4}$ on his particle 4,

$$
U_{4}|\varphi\rangle_{64}=|\phi\rangle_{64} \text { where } U_{4}=\left(\frac{\bar{D} B \bar{F} C}{\rho_{1} \rho_{2}}\right)^{-1}
$$

,Bob captured the state $|\phi\rangle$. We find that Bob's local operator is independent with the state $|\phi\rangle_{12}$.

\section{Conclusion}

After introduced a new notation for the quantum information, we obtained a criteria for pure bipartite entangled state and a theorem for bipartites quantum teleportation by two EPR pairs. We constructed the operation $U$ performed by the $B o b$ on one of his qubit in order to obtain perfect replica of teleported states.

\section{References}

[1] A. Einstein, B. Podolosky and N. Rosen, Phys. Rev. 47777 (1935).

[2] C. H. Bennett and S. J. Wiesner, Phys. Rev. Lett. 69 ,2881 (1992).

[3] M. Zukowski, A. Zeilinger, M. A. Horne, and A. K. Ekert,Phys. Rev. Lett. 71, 4287 (1993)

[4] C. H. Bennett et al. Phys. Rev. Lett. 701895 (1993).

[5] L. Vaidman, Phys.Rev. A 491473 (1994).

[6] S. L. Braunstein and H. J. Kimble, Phys. Rev. Lett. 80869 (1998).

[7] S. Bose and V. Vedral, Phys. Rev. A 61, 040101(2000).

[8] S. L. Braunstein, G. M. D'Ariano, G.L. Milburn and M. F. Sacchi, Phys.Rev.Lett. 843486 (2000).

[9] L. Accardi and M. Ohya, Quantum information Proceedings of the First International Conference Meijo University, Japan 4 - 8 November 1997 
[10] L. P. Hughston, R.J. Ozsa and W.K. Wootters, Phys. Lett. A 18314 (1993)

[11] C.Li,H.S.Song and Y.X.Luo,Phys. Lett. A 297 (2002) 121

[12] J.Lee and M.S. Kim,Phys. Rev.Lett.84 (2000)4236

[13] X.Wang, Phys. Rev.A64 (2001)022302

[14] J. Lee, H. Min and S.D. Oh, Phys.Rev. A 66 (2002)052318 
This figure "1.jpg" is available in "jpg" format from: http://arxiv.org/ps/quant-ph/0303098v1 